\title{
ASSESSMENT OF IRIS NEOPLASMS *
}

\author{
BY \\ FRANK W. LAW AND PETER HANSELL \\ London
}

THE following case history is reported not only as having some intrinsic clinical interest but also because it illustrates the possible value of a photographic method of checking an increase in the size of a neoplasm of the iris or other small tumour.

\section{Case Report}

A man aged 42 was first seen in March, 1950. A "blemish" had been noticed on the right iris 7 years before, and on his recent return to the surgeon who had remarked upon this, the latter gave the opinion that it had increased in size, and recommended a second opinion and surgical removal of the lesion.

Examination.-A pigmented mass on the right iris at 4 o'clock measured 2.5 by $2 \mathrm{~mm}$., the long axis being radially placed. It just overlapped the pupillary margin and was well raised above the surface, and the surrounding normal iris came up to the main mass as "foothills". The surface had the typical pile of a melanoma; no distortion of the iris was visible, and there was nothing to be seen on the posterior surface of the iris, which appeared flat.

The lesion was regarded as of potentially sinister significance, but immediate removal was not advised. A photograph was taken (Fig. 1) and the patient was asked to return in 3 months.

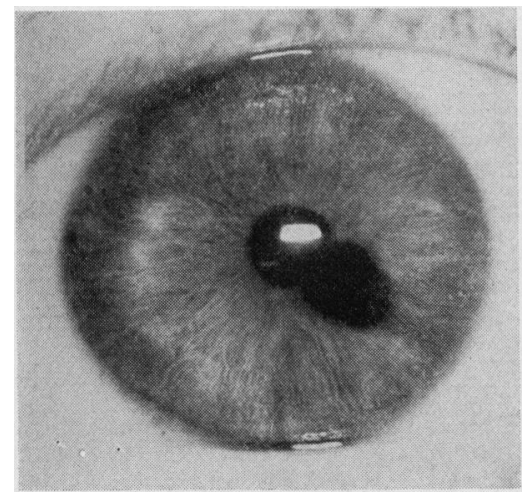

FIG. 1.-One picture from a stereoscopic pair taken with a fixed-focus camera. $(\times 4$.) Original record taken March, 1950.

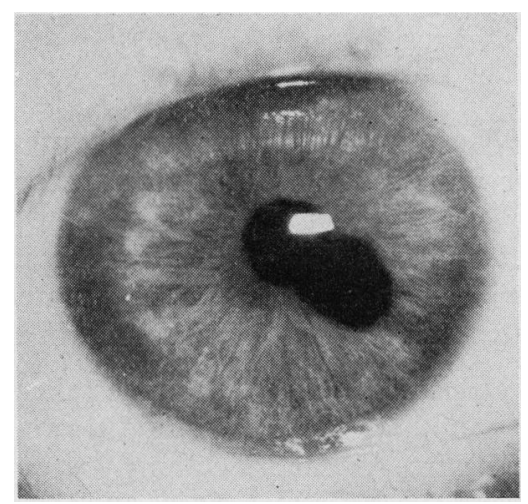

Fig. 2.-Comparative record taken February, 1952. ( $\times 4$.

* Received for publication, June 26, 1956 
He appeared again 2 years later (1952), but only because he had lost his glasses! The appearance was unchanged; a small area of gelatinous appearance with slight pigmentation which had been previously noted at the limbus at 7 o'clock, and which can be detected in the photograph, was also unchanged. A second photograph was taken (Fig. 2) and a third another year later (Fig. 3, 1953), but no change was detectable in either.

In November, 1955, it was possible to see a small swelling on the posterior surface of the iris by very oblique illumination; an increase in size was suspected. The lesion was re-photographed as a routine (Fig. 4) and comparison with previous records revealed a small but definite increase in size.

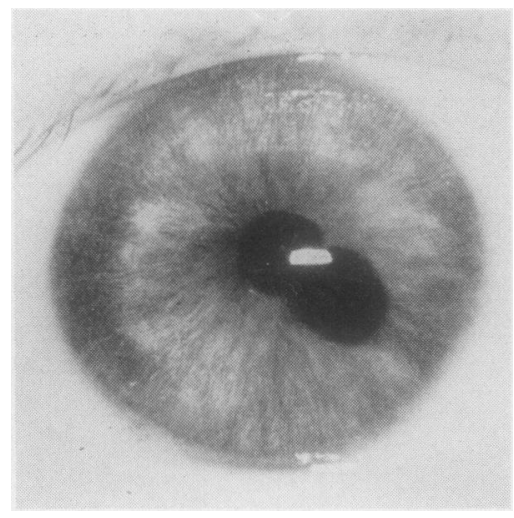

Fig. 3.-Further comparative record taken April, 1953. ( $\times 4$.)

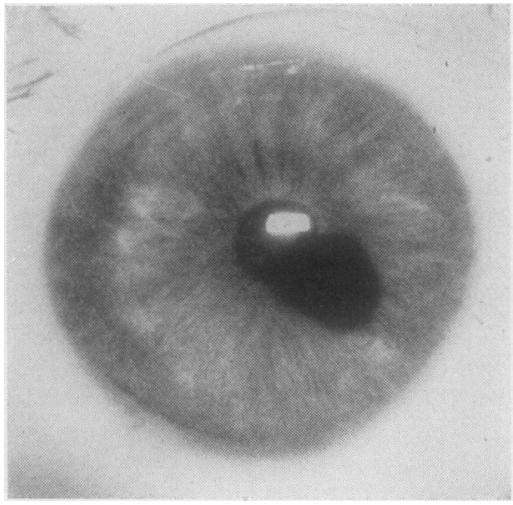

FIG. 4.- Record taken in September, 1955. This is the first to show a measurable change since the original photograph. ( $\times 4$.$) Compare visually with$ Figs 1 and 3.

Thus, some four carefully controlled photographic records had been made of the patient's iris. In each instance comparison with previous records was made by visual superimposition of the negatives-a crude but effective method of detecting two-dimensional changes. Viewed in this way, the first three records (embracing a 3-year period) matched perfectly, whereas the fourth showed a calculated increase of $0.25 \mathrm{~mm}$. in the diameter of the lesion (Fig. 5, opposite).

Operation.-This was undertaken in January, 1956, under general anaesthesia. A conjunctival flap was laid down, and the globe was opened by keratome and scissors. A wide iridectomy was performed extending to the iris root; two corneoscleral sutures were placed, and the conjunctival flap sutured. The iris was closely adherent to the lens above, and a localized opacity was present. The eye recovered normally (Fig. 6, opposite). Gonioscopy confirmed the presence of synechiae at 3 o'clock, and some distortion of the ciliary processes was seen at 6 o'clock.

A high astigmatism, due to the operation, gradually disappeared, and corrected vision 4 months later was $6 / 5$. 


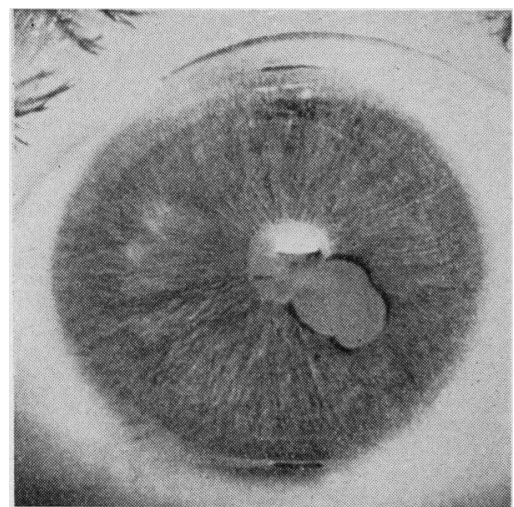

FIG. 5.-For the purpose of demonstration, the picture shewn in Fig. 1 has been reversed and superimposed on the negative of Fig. 4. The dark halo surrounding the tumour therefore represents the increase in size. $(\times 4$.

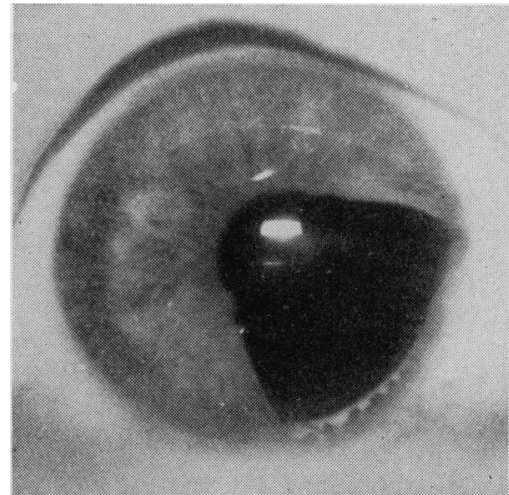

FIG. 6.-Post-operative photograph for comparison. $(\times 4$.

Histologica! Report (from the Department of Pathology, Institute of Ophthalmology).-This confirmed the diagnosis of melanoma and suggested malignancy. It stated that no myoglial fibres were present, and added the disturbing suggestion that excision had been incomplete, since heavily pigmented naevoid cells extended to the limit of the excision (Fig. 7).

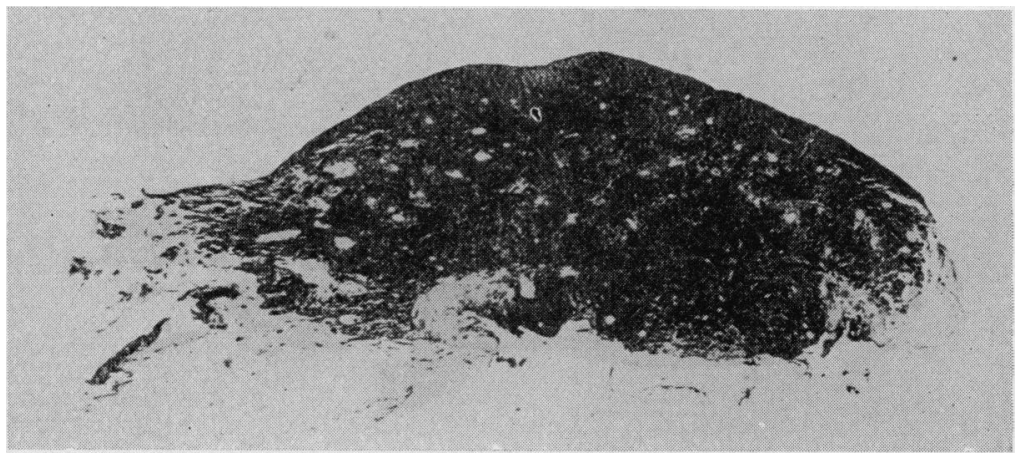

FIG. 7.-First section of entire lesion. $(\times 30$. $)$

This finding, which is also illustrated by Figs 8 and 9 (overleaf), was so surprising in view of the width of the iridectomy that further sections were requested. It was then found that folding had occurred, probably because the specimen was imperfectly spread before being pinned out upon cork immediately after excision. Remounting and further sections showed that excision had been complete (Fig. 10 , overleaf). 


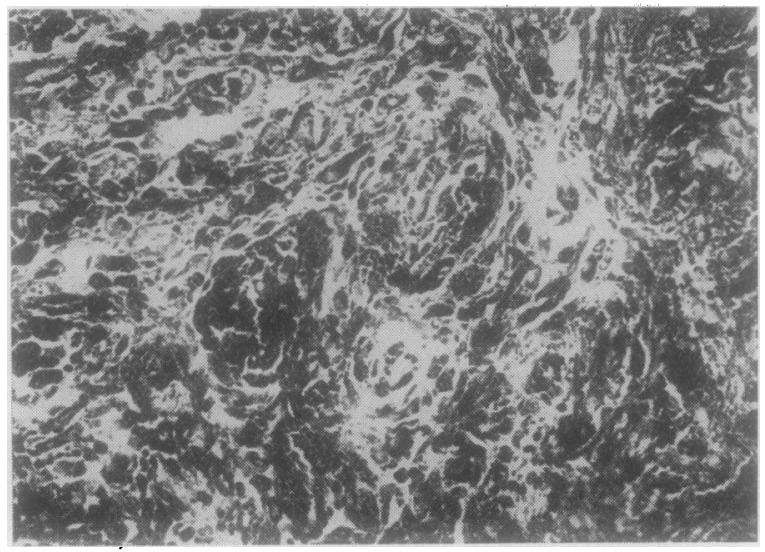

FIG. 8.-High-power view of tumour. $(\times 250$. $)$

FIG. 9.-Bleached section for comparison with Fig. 8 . $(\times 250$.)
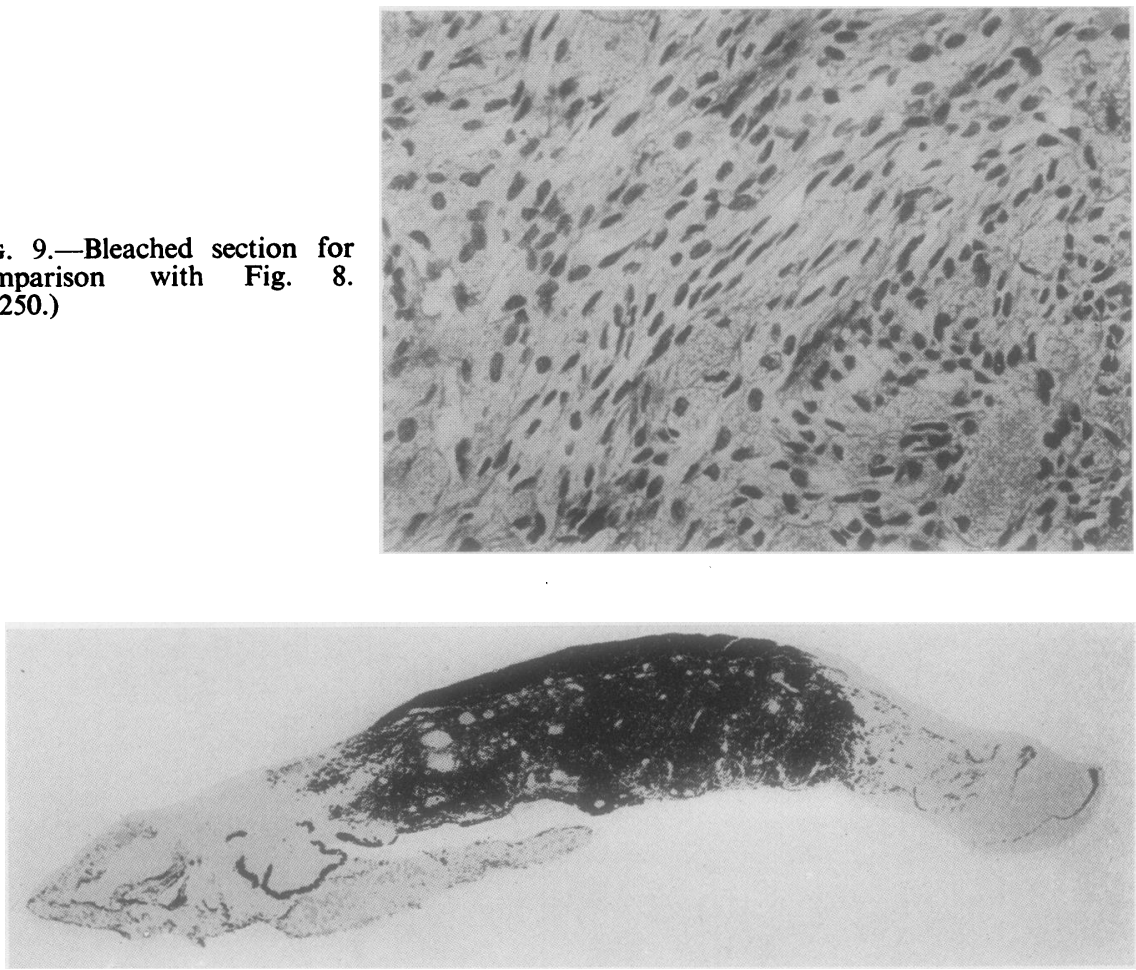

FIG. 10.-Second section showing complete removal of growth. $(\times 24$. $)$

An interesting feature of this section is the presence of what appears to be lens capsule, to be seen clear of the growth, at the left-hand end of the section, which caused immediate qualms lest the lens opacity observed at operation should spread. This has not occurred up to the present. 


\section{Discussion}

It is reasonable to suppose that, in the absence of obvious growth, or distortion of surrounding tissues, clinical evaluation of small melanomata over a period of time is not always reliable. It would be unrealistic to depend on visual memory to detect a dimensional change of even $0.5 \mathrm{~mm}$. spread over a few years.

This case provides the opportunity for the description of a method which may well prove important in the objective determination of tumour growth. This seems to offer certain advantages over subjective clinical impression when small and slowly growing neoplasms are under periodic observation. No special apparatus is required and strict attention to working methods will ensure an accuracy upon which the clinician can depend.

Photographic negatives made on glass by means of a fixed-focus camera provide records of no mean accuracy. In detecting small changes, some magnification at the negative stage is desirable, but otherwise a standard magnification of four or more times can be achieved in the enlarger; this obviously facilitates measurement.

The photographic aspects of this problem could be elaborated indefinitely, depending on the precision required. Suffice it to say that superimposition of carefully scaled negatives taken at a certain magnification undoubtedly provides the quickest visual-cum-photographic assessment of changes. It is further suggested that stereophotogrammetric methods offer accuracy to within $0.01 \mathrm{~mm}$. or less, and include the third dimension. Other principles are involved, however, and work is still proceeding along these lines.

\section{Summary}

A case of melanoma of the iris, which was removed surgically, is described, including a photographic method of observing and verifying any increase in size of such lesions. 